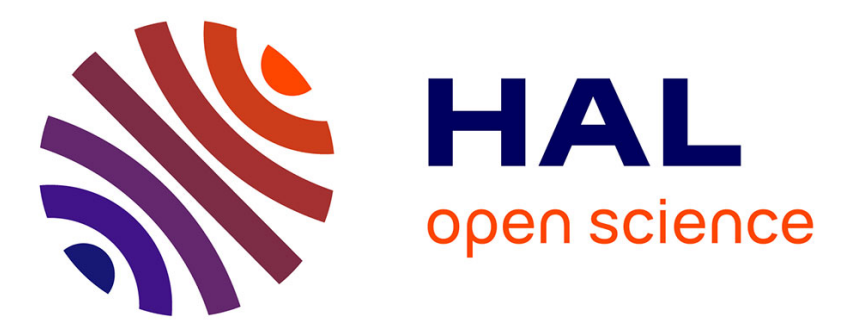

\title{
Photoacoustique, photothermique et télécommunications
}

\author{
D. Fournier, A.C. Boccara, F. Lepoutre, J. Badoz
}

\section{To cite this version:}

D. Fournier, A.C. Boccara, F. Lepoutre, J. Badoz. Photoacoustique, photothermique et télécommunications. Revue de Physique Appliquée, 1984, 19 (2), pp.59-62. 10.1051/rphysap:0198400190205900 . jpa-00245173

\section{HAL Id: jpa-00245173 https://hal.science/jpa-00245173}

Submitted on 1 Jan 1984

HAL is a multi-disciplinary open access archive for the deposit and dissemination of scientific research documents, whether they are published or not. The documents may come from teaching and research institutions in France or abroad, or from public or private research centers.
L'archive ouverte pluridisciplinaire HAL, est destinée au dépôt et à la diffusion de documents scientifiques de niveau recherche, publiés ou non, émanant des établissements d'enseignement et de recherche français ou étrangers, des laboratoires publics ou privés. 


\title{
Photoacoustique, photothermique et télécommunications
}

\author{
D. Fournier, A. C. Boccara, F. Lepoutre et J. Badoz \\ Laboratoire d'Optique Physique, ESPCI, 10, rue Vauquelin, 75231 Paris Cedex 05, France
}

(Reçu le 3 juin 1983, révisé le 19 septembre, accepté le 26 octobre 1983)

\begin{abstract}
Résumé. - Nous décrivons les principales applications des détections photoacoustiques et photothermiques d'une part au contrôle non destructif des matériaux et de l'environnement, d'autre part à la caractérisation des composants optiques ou électroniques et à l'imagerie.
\end{abstract}

Abstract. - The main applications of photoacoustic and photothermal detection are described for non destructive testing of materials and environment, characterization of optical and electronical devices and imaging.

La photoacoustique (ou optoacoustique) est née accidentellement, il y a à peine plus de cent ans, au cours d'expériences de télécommunications optiques effectuées par A. G. Bell [1].

Pour engendrer un signal « sonore " à partir d'une source lumineuse, il suffit d'irradier, à l'aide d'un faisceau lumineux modulé ou pulsé, un échantillon susceptible d'absorber l'énergie lumineuse incidente, placé dans une cellule fermée. L'énergie lumineuse absorbée provoque l'échauffement et, par suite, la dilatation de l'échantillon. Celui-ci va transférer une part de son énergie thermique au milieu transparent dans lequel il baigne. Cet effet représente la contribution principale à la variation de pression du gaz contenu dans la cellule [2].

On appelle signal photoacoustique, le signal dépendant du temps issu du microphone qui mesure les variations de pression de la cellule. C'est une mesure indirecte de l'échauffement de l'échantillon et, par suite, de son coefficient d'absorption optique et de ses paramètres thermiques. On peut procéder aussi à cette mesure en collant l'échantillon sur un détecteur piézoélectrique : les variations de volume engendrées par l'absorption de l'énergie lumineuse modulée ou pulsée permettent d'atteindre les mêmes paramètres.

Les principales applications de la détection photoacoustique du rayonnement qui touchent à la spectroscopie et au contrôle non destructif, se situent dans des domaines très variés : physico-chimie, biologie, mesures thermiques, détection de polluants atmosphériques, imagerie...

Notons enfin que la détection "acoustique " n'est pas la seule employée et que l'on peut souvent mesurer directement avec une meilleure sensibilité, l'élévation de température photo-induite. L'utilisation d'un fais-

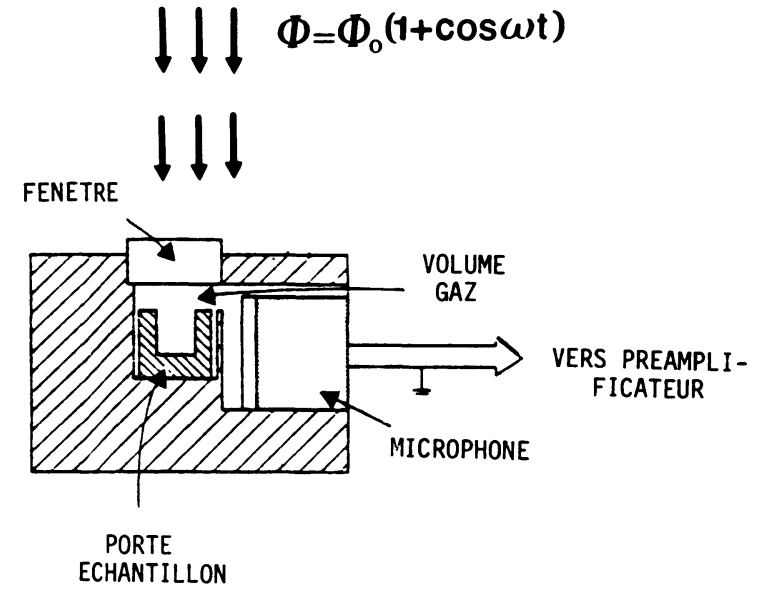

Fig. 1. - Schéma d'une cellule photoacoustique : un flux lumineux modulé à la fréquence $\omega / 2 \pi$ est absorbé par l'échantillon qui est ainsi chauffé. Un transfert de chaleur a ensuite lieu entre l'échantillon et le gaz causant une variation périodique de pression mesurée par le microphone.

[Photoacoustic cell scheme : the light beam modulated at $\omega / 2 \pi$ frequency, is irradiating the sample which is so heated. The heat transfered to the gas induces a periodic pressure measured by a microphone.]

ceau sonde dont la déviation est liée au gradient de température dans la zone chauffée (effet " mirage "), mise au point dans notre laboratoire [3] permet un gain en sensibilité de plusieurs ordres de grandeur (Fig. 2).

Nous essaierons de montrer dans cet exposé quels peuvent être les rapports de cette technique avec le domaine des télécommunications : c'est l'aspect contrôle qui sera principalement mis en jeu pour les 


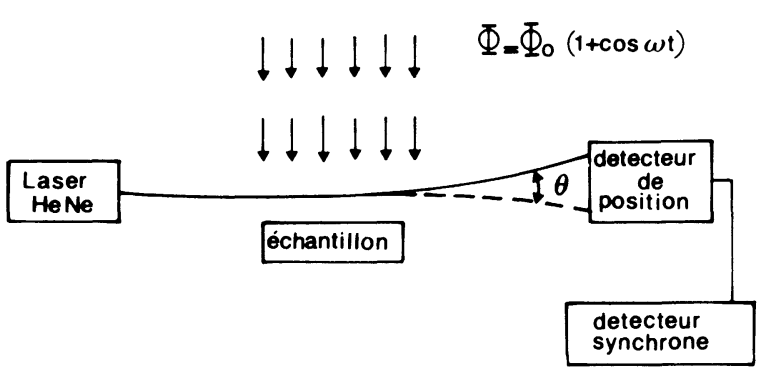

Fig. 2. - Principe de la détection par effet "mirage". C'est la diffusion thermique à l'interface entre l'échantillon et le fluide environnant (gaz ou liquide) qui est à l'origine $\mathrm{du}$ gradient de température produisant la déviation du faisceau sonde.

[Detection by " mirage effect ": the probe beam is deflected in going through the heated area near the sample.]

«matériaux " ainsi que pour les «composants » optiques ou électroniques.

\section{Contrôle des matériaux et de l'environnement.}

Directement dérivé des études spectroscopiques, ce contrôle peut toucher de nombreux domaines liés à la mise au point ou à la fabrication de matériaux pour les télécommunications. Nous évoquerons ici quelques points qui nous paraissent importants concernant les matériaux semiconducteurs, les lasers et les gaz toxiques.

\section{- Semiconducteurs.}

De nombreux matériaux semiconducteurs, cristallins ou amorphes, sont aujourd'hui caractérisés par une étude de l'absorption optique dans la bande interdite : cette méthode, qui permet de mesurer la densité de centres profonds ou de liaisons non saturées, ne peut être menée à bien par les techniques spectrophotométriques traditionnelles, car les signaux dus à cette faible absorption sont masqués par les phénomènes de réflexion ou d'interférence lorsque les matériaux sont préparés sous forme de couches minces. La méthode photothermique (" effet mirage ») échappe à ces problèmes puisque le signal est proportionnel seulement à la densité d'énergie absorbée dans le matériau (Fig. 3). Il existe, à notre connaissance, une vingtaine de groupes universitaires ou industriels (Xerox, RCA, EXXON...) qui utilisent aujourd'hui la méthode " mirage " mentionnée plus haut que nous avions introduite dans le but de caractériser le silicium amorphe [4]. Notons également que l'observation peut avoir un caractère local et qu'il est possible de contrôler le dopage, la cristallinité et même le recuit in situ en utilisant le même laser pour engendrer le signal et effectuer le recuit [5].

- Matériaux pour lasers.

Puisque les méthodes décrites permettent d'accéder à la quantité d'énergie thermique déposée dans les échantillons, elles ont pu être utilisées avec succès pour mesurer les rendements quantiques de maté-

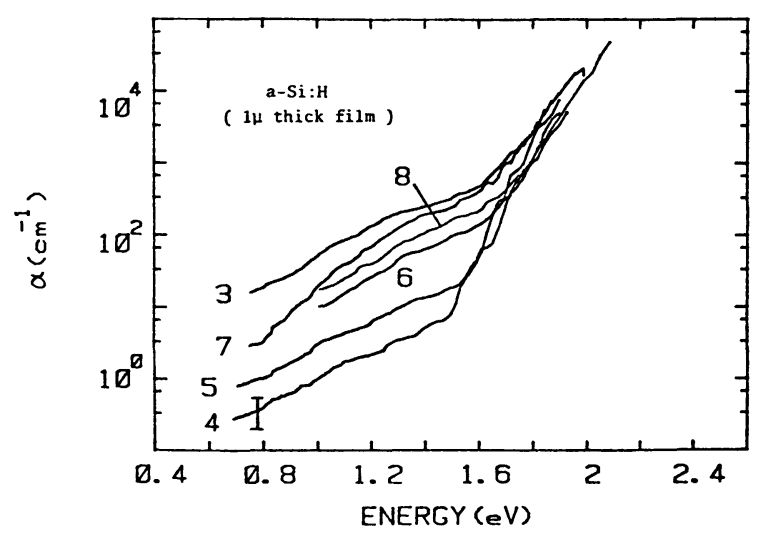

Fig. 3. - Absorption de différents échantillons de silicium amorphe dans la bande interdite (référence 4).

[Absorption of different samples of amorphous silicon in the band-gap (Ref. 4).]

riaux destinés à la fabrication de lasers pour l'optique intégrée [6].

Notons également que la même méthode peut être utilisée pour optimiser les rendements de photodétecteurs, en minimisant les pertes par effet Joule.

- Contrôle in situ de gaz toxiques.

De nombreux composants sont aujourd'hui préparés ou dopés à partir de gaz (ex. : silane, borane, germane...) dont certains sont dangereux, et il paraît nécessaire de disposer en milieu industriel de méthodes in situ permettant une détection simple et sensible des gaz toxiques.

Nous avons proposé [7] de mesurer directement dans l'air l'échauffement produit par un laser grâce à la déviation d'un laser sonde qui se propage dans la zone chauffée. Sans qu'aucun prélèvement de gaz

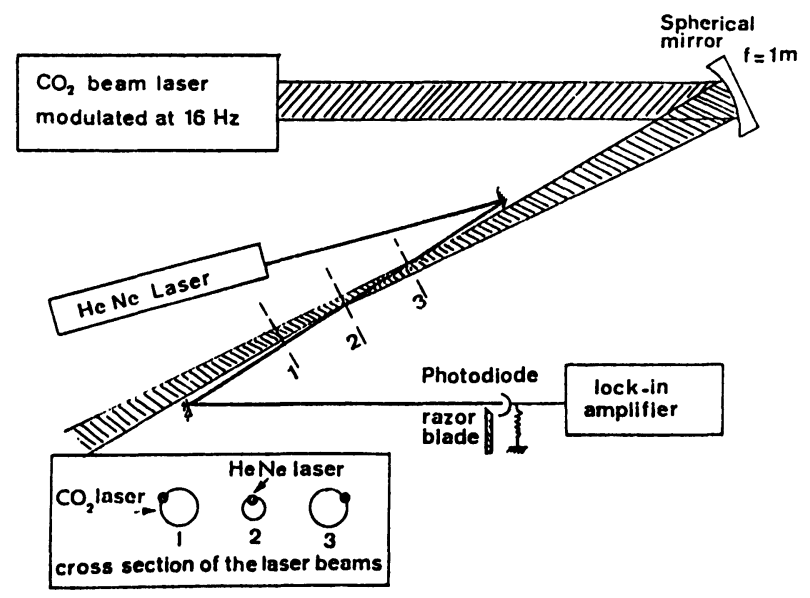

Fig. 4. - Contrôle in situ de polluants atmosphériques. Le faisceau sonde est dévié dans la zone de gradient de température produite par absorption du laser à $\mathrm{CO}_{2}$ (référence 7).

[Detection in situ of atmospheric pollution. The probe beam is deviated in the area of the temperature gradient due to the absorption of the $\mathrm{CO}_{2}$ laser beam.] 
ne soit nécessaire, une sensibilité correspondant à un seuil de détectivité de l'ordre du ppb a pu être obtenue pour l'éthylène soumis à une irradiation de $1 \mathrm{~W}$ (Raie $\mathrm{P}_{14}$ à $10,4 \mu$ ) (Fig. 4).

\section{Contrôle des composants et imagerie photother- mique.}

- Composants optiques.

Les mesures photothermiques apparaissent comme des méthodes intéressantes pour compléter les mesures habituelles de pertes dans les guides d'ondes optiques.

Dans le cas des fibres, ces méthodes permettent de séparer la contribution de l'absorption du matériau de celle de la diffusion. La sensibilité peut être de l'ordre du $\mathrm{dB} / \mathrm{km}$ et pourra sans doute être améliorée dans l'avenir [8].

Pour ce qui est des "slabs" utilisés en optique intégrée, il est possible, avec le schéma de la figure 5, de contrôler non seulement les pertes dans les guides, mais d'optimiser le couplage avec un laser extérieur en maximisant le signal d'effet mirage, c'est-à-dire l'énergie lumineuse présente dans le guide.

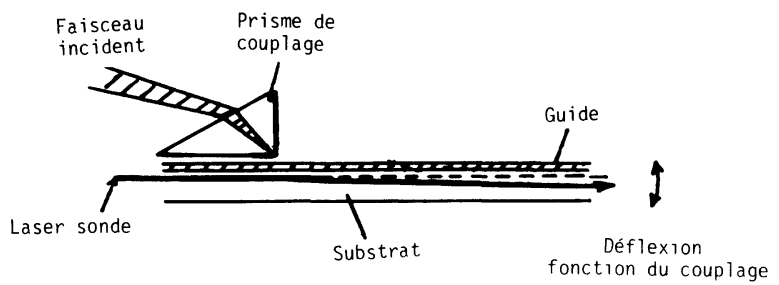

Fig. 5. - Mesure des pertes par absorption ou du couplage guide d'onde-laser par déflexion d'un faisceau sonde se propageant dans le substrat.

[Absorption losses and wave-guide laser coupling measurements by diffusion of a probe beam propagating in the substrat.]

\section{- Composants électroniques et imagerie photother-} mique.

C'est vers ce domaine que se focalisent aujourd'hui les recherches qui utilisent l'imagerie photothermique. Rappelons que l'irradiation périodique de l'échantillon à l'aide d'un faisceau lumineux ou d'un faisceau d'électrons, permet la création d'« ondes thermiques »

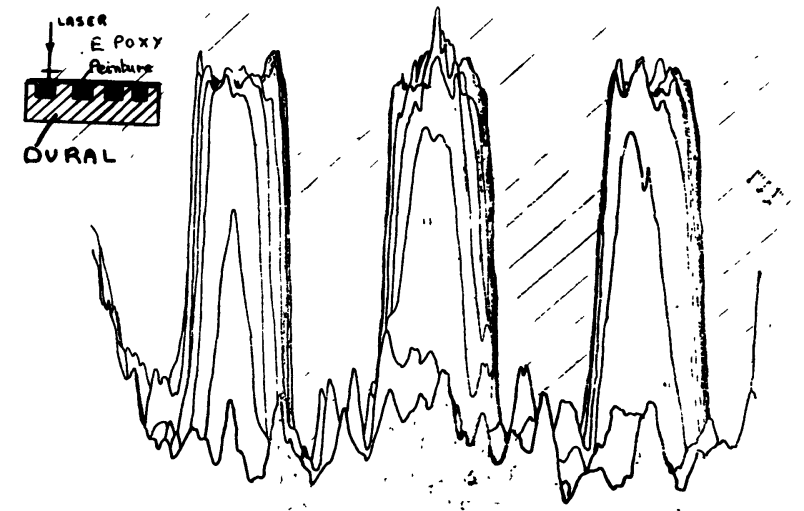

Fig. 6. - Mise en évidence d'hétérogénéités thermiques invisibles optiquement. Balayage point par point.

[Detection of thermal heterogeneities in a sample.]

qui seront utilisées comme sonde de la matière puisque, à une échelle qui est celle de la longueur de diffusion thermique, on peut observer leur réflexion, leur transmission ou leur diffraction, et détecter la présence de défauts ou de structures invisibles optiquement, masquées sous la surface de l'échantillon. Le signal représenté sur la figure 6 est le résultat d'une expérience préliminaire montrant la détection de défauts présentant des propriétés thermiques différentes de celles du substrat, cachées sous une couche de peinture noire.

Nous voudrions souligner les résultats obtenus dans le domaine de la microélectronique par A. Rosencwaig. Le couplage d'une irradiation constituée par un flux d'électrons modulé et d'une détection piézoélectrique permet en effet de détecter des défauts, à l'échelle submicronique, qui ne sont pas observables sur l'image obtenue directement à l'aide d'un microscope électronique. L'image " thermique » permet en effet d'accéder à des défauts d'adhésion subsurfaciques, des défauts de cristallinité (joints de grain) et des défauts de dopage [9]. La figure 7 représente d'une part l'image en microscopie électronique classique, et d'autre part les images "thermiques » $(b)$ et $(c)$, enregistrées à deux phases différentes (ce qui revient à effectuer une observation à deux profondeurs dif-

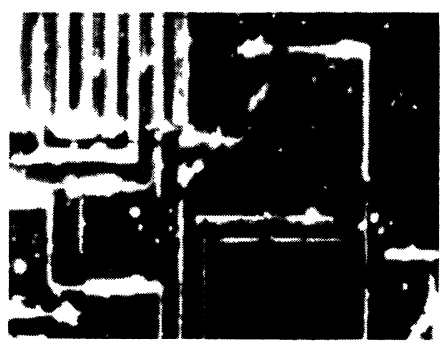

(a)

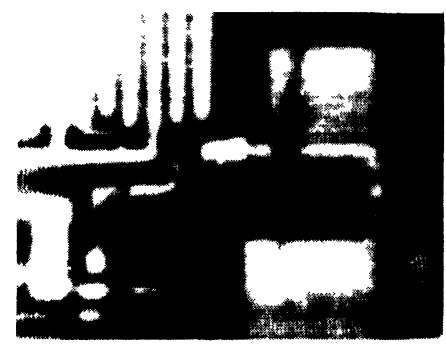

(b)

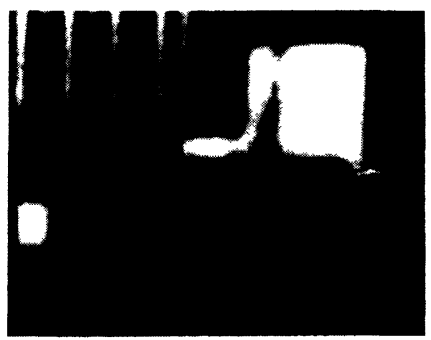

(c)

Fig. 7. - Image d'un circuit intégré en microscopie électronique $(a)$ et en ondes thermiques excitées par un faisceau d'électrons pour une observation à $1 \mu(b)$ et à $3 \mu(c)$ sous la surface (référence 10), avec la permission de A. Rosencwaig.

[Integrated circuit imaging : electron micrograph (a), and thermal-wave imaging $(b, c)$ with two different phases allowing two depth profiling (with the permission of A. Rosencwaig).] 


\section{section a a}

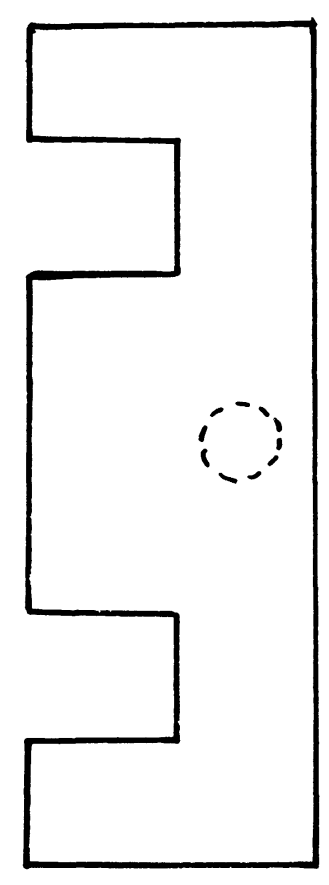

a

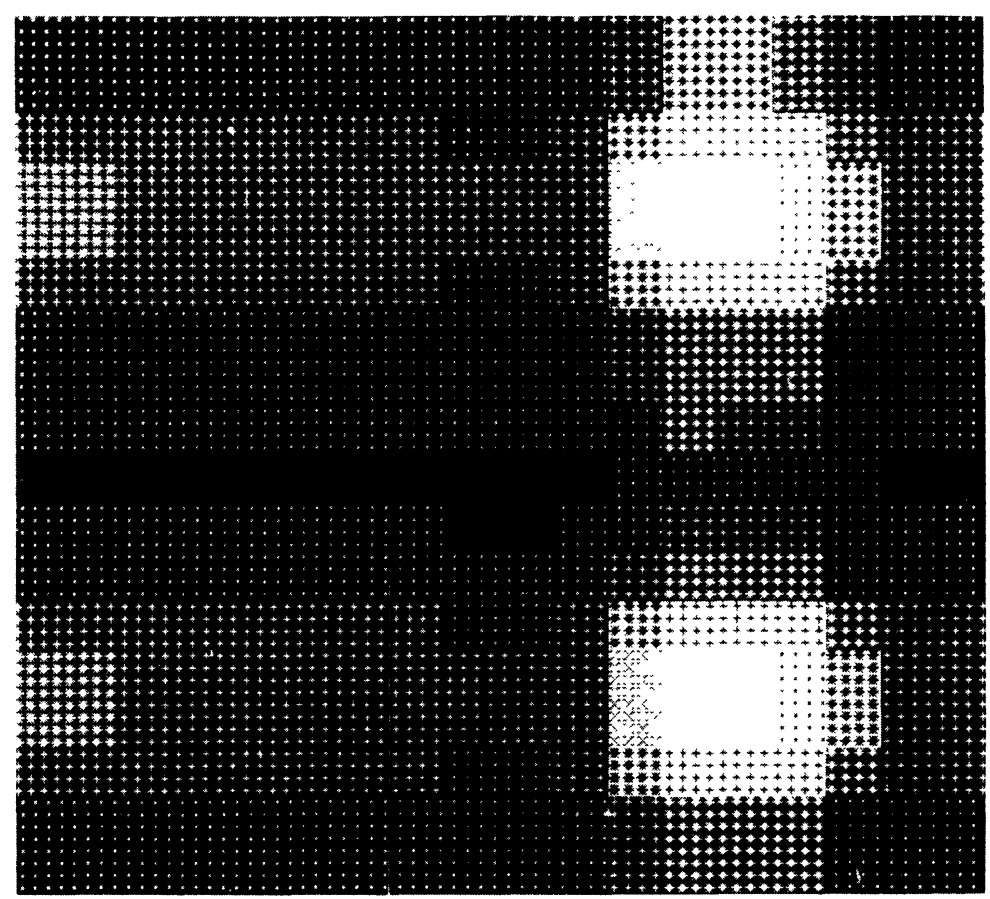

a

Fig. 8. - Mise en évidence de défauts subsurfaciques; approche tomographique : balayage ligne par ligne.

[Detection of subsurface defects : tomographic approach : line by line scanning.]

férentes) et permettant de mettre en évidence un défaut d'adhésion à $3 \mu$ de la surface environ [10].

Nous avons, pour notre part, récemment utilisé l'effet " mirage " avec un balayage ligne par ligne et un dépouillement du type tomographique des résultats obtenus (Fig. 8). Ce type d'expériences permet de mettre en évidence, très rapidement, la présence de défauts tout en réduisant la densité d'énergie sur l'échantillon.

Bien que les détections photoacoustiques et photothermiques n'aient été employées que très récemment pour le contrôle non destructif, il nous semble qu'elles tendent à s'imposer rapidement à côté d'autres méthodes plus connues (ultrasons, imagerie X...). Elles présentent souvent une spécificité liée aux processus mêmes de diffusion de la chaleur qui en font un nouvel outil présentant des possibilités remarquables. Les progrès récents des méthodes de calculs des effets thermomécaniques dans les milieux hétérogènes ainsi que les développements instrumentaux qui visent à une augmentation de la sensibilité, contribueront à rendre ces méthodes quantitatives opérationnelles.

\section{Bibliographie}

[1] Bell, A. G., Ann. J. Sci. 20 (1880) 305.

[2] Rosencwaig, A. and Gersho, A., J. Appl. Phys. 47 (1976) 64.

[3] Boccara, A. C., Fournier, D. and Badoz, J., Appl. Phys. Lett. 36 (1980) 130.

[4] Jackson, W., Amer, N., Fournier, D. and Boccara, A. C., Technical Digest, 2nd int. meeting on Photoacoustic spectroscopy, Berkeley (1981).

[5] McClelland, J. F. and Kniseley, R. N., Appl. Phys. Lett. 35 (1979) 585.
[6] Auzel, F., Meichenin, D. and Michel, J. C. (1978), Proc. Int. Conf. on Luminescence, Paris 1978, North Holland Amsterdam.

[7] Fournier, D., Boccara, A. C., Amer, N. and Gerlach, R., Appl. Phys. Lett. 37 (1980) 519.

[8] Huard, S. and Chardon, D., Opt. Commun. 39 (1981) 59.

[9] Rosencwaig, A., Electron. Lett. 19 (1980) 928.

[10] RosenCwaig, A., Solid State Techn. (1982) 1981. 